\title{
Low Intensity Laser as an Adjuvant to Improve Muscle Strength after Exercise: A Case Report
}

\author{
Hélio Plapler ${ }^{1}$, André Tadeu Sugawara ${ }^{2 *}$, Rita de Cássia Montelli ${ }^{3}$ and Linamara Rizzo \\ Battistella ${ }^{4}$
}

${ }^{1}$ Full Professor, Department of Surgery, Paulista School of Medicine, Federal University of São Paulo, São Paulo, Brazil ${ }^{2}$ Physiatrist, Laboratory of Isokinetic, Institute of Medicine and Rehabilitation, Faculty of Medicine of the Hospital of Clinics of São Paulo, São Paulo, Brazil

${ }^{3}$ Physiotherapist, Laboratory of Isokinetic, Institute of Medicine and Rehabilitation, Faculty of Medicine of the Hospital of Clinics of São Paulo, São Paulo, Brazil

${ }^{4}$ Full Professor, Head of Division, Institute of Medicine and Rehabilitation, Faculty of Medicine of the Hospital of Clinics of São Paulo, São Paulo, Brazil

\begin{abstract}
Muscle fatigue is a limiting factor in prosthetic patients and studies show that low intensity laser can improve performance and increase muscle strength. We tested a concentric exercise protocol comparing a test applied after 10 sessions without and with the use of laser using the Cybex Humac Norm ${ }^{\circledast}$ Test \& Rehabilitation System in a patient with left lower limb amputation. First, a test was performed to determine the baseline value of peak torque, average power per repetition and total work performed. Ten exercise sessions and a new test were followed to determine the difference in power gain. Ten more sessions and a new test without laser application were performed to determine whether the torque increment level was reached, followed by ten more sessions with laser application 5 minutes before the exercise and a new test, with laser application, to evaluate the torque increment. An increase in PT was found of $5 \%$ for extensors and $7 \%$ for knee flexors in the second test in relation to baseline. The increase was $0 \%$ for the extensors and $11 \%$ for the flexors in the next test. After laser application there was an increase of $8 \%$ for the extensors and a deficit of $11 \%$ for the flexors. For TTR, the relative values for extenders and flexors were $3 \%$ and $-3 \%,-10 \%$ and $-2 \%$ (without laser) and $18 \%$ and $17 \%$ (with laser). We concluded that, in this protocol, the laser produced a gain in the peak torque of extensor muscles, but not of the flexors as well as a significant increase in the average power per repetition and the total work performed by both the extensors and the knee flexors, which may be of value as an adjuvant in the treatment of prosthetic patients.
\end{abstract}

\section{Keyword}

Low-level light therapy, Muscle fatigue, Muscle strength, Human

\section{Introduction}

Prosthetic patients, especially those of older age, give up the use of the prosthesis mainly due to fatigue due to exertion, causing loss of quality of life and waste of resources in rehabilitation and distribution of these equipment. Muscle fatigue in anaerobic exercises is caused, in part, by the production of reactive oxygen species (ROS) [1] impairing mitochondrial function, which causes the depolarization of muscle cells, decreasing their ability to generate strength. Among the various ways to reduce fatigue, the application of low intensity laser (LIL) is promising but is still little known. Research has shown that the application of LIL in different protocols was able to increase the number of contractions and promote decreased creatine kinase (CK) activity or at least to promote a lower increment of muscle proteins activity in blood [2,3]. Another study showed that photobiomodulation with LIL associ- ated with an exercise program was able to improve postural stability and decrease the risk of falling in elderly patients [4]. One of the ways to promote muscle fatigue is through repeated contraction exercises with maximum endurance (isokinet-

*Corresponding author: André Tadeu Sugawara, Physiatrist, Laboratory of Isokinetic, Institute of Medicine and Rehabilitation, Faculty of Medicine of the Hospital of Clinics of São Paulo, São Paulo, Rua Domingo de Soto, 100 CEP: 04116-030, Tel: 55(11)-5180-7800, Brazil

Accepted: December 29, 2020

Published online: December 31, 2020

Citation: Plapler H, Sugawara AT, Montelli RC, et al. (2020) Low Intensity Laser as an Adjuvant to Improve Muscle Strength after Exercise: A Case Report. J Phys Med 2(1):55-59 


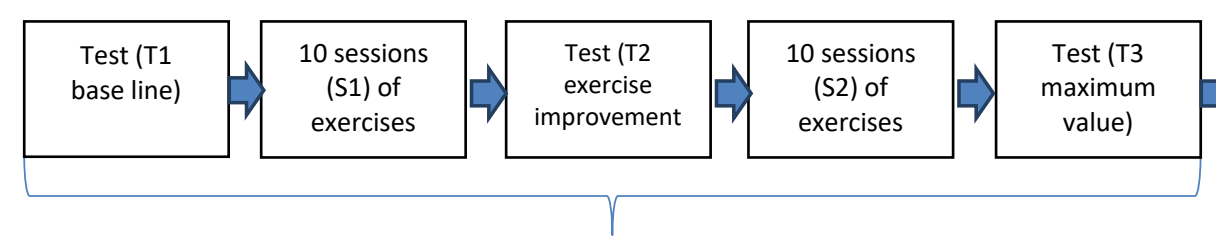

Without laser

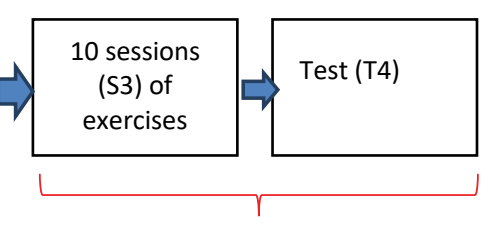

with laser / sham

Figure 1: Design of the test protocol and exercise sessions.

Table 1: Protocol of each exercise session on the Cybex Humac Norm device.

\begin{tabular}{|l|l|l|l|l|l|l|l|l|l|l|}
\hline Angular velocity $(\% / \mathrm{s})$ & 60 & 90 & 180 & 240 & 300 & 270 & 210 & 150 & 90 & 60 \\
\hline Number of repetitions & 6 & 8 & 13 & 16 & 19 & 17 & 14 & 12 & 8 & 6 \\
\hline
\end{tabular}

ic exercises). Through this type of exercise, it is possible to measure the peak torque, power and total work produced by a muscle group. In neuromuscular training, improving physical-functional performance by minimizing muscle fatigue can bring benefits in the recovery specially in prosthetic patients, in which intense muscular work is required. In this scenario, we tested the action of LIL to decrease muscle fatigue in a patient in a rehabilitation program after total lower limb amputation.

\section{Case Presentation}

A 67-year-old male patient with cervical skin cancer metastasis underwent lymphatic emptying in the left cervical region followed by radiotherapy in 2015. He evolved with bone metastasis in the upper and lower limbs, underwent a titanium rod implantation in the left femur and chemotherapy. With the evolution of the tumor in the left lower limb, reaching the muscles of the gluteal region, he underwent amputation of the left lower limb and enlarged hip disarticulation (hemipelvectomy) in December 2017. He began a physiotherapy program in March 2018 and received complete left lower limb prosthesis in June 2018. At the beginning of treatment, he was $178 \mathrm{~cm}$ tall and $60 \mathrm{~kg}$ in weight with $8.5 \%$ fat measured by bioimpedance, BMI = 19. In January 2019 he was weighing $69 \mathrm{~kg}$.

\section{Exercise protocol}

This study was included in Brazil Platform under the number 4.040 .364 and approved by the Ethics Committee under the number 4.196.253. The protocol design is shown in (Figure 1). The patient was first submitted to a test using a CYBEX Humac Norm ${ }^{\circledR}$ dynamometer (CSMI, Stoughton). First, the patient was asked to make 3 free extension/flexion movements as heating. Then, he performed a set of 5 repetitions of concentric knee extension/flexion movements at an angular velocity of $60^{\circ} . \mathrm{s}^{-1}$ to evaluate the peak torque (PT). After a one-minute rest, 5 more repetition movements were performed at the angular velocity of $60^{\circ} . \mathrm{s}^{-1}$, after another one-minute interval, 30 repetitions were performed with an angular velocity of $300^{\circ} . \mathrm{s}^{-1}$ for the evaluation of the total work performed (TWP). These values were considered baseline values. He was then submitted to 10 sessions of exercise, twice a week, as described below (Table 1). After these sessions, a new test was performed as described above. The laser was not applied before the exercise sessions or before the test. These values were considered as an increase due to the exercises.

Ten more exercise sessions were performed twice a week applying the low intensity laser (DMC Ltd, São Carlos, Brazil), at 4 equidistant points along the longitudinal axis of the anterior and the posterior face of the thigh, with a wavelength of $660 \mathrm{~nm}$, continuous mode, nominal power $100 \mathrm{~mW}$, frequency of $50 \mathrm{~Hz}$, spot diameter of $2 \mathrm{~mm}$, power density $32.2 \mathrm{~W} /$ $\mathrm{cm}^{2}$.per point, irradiation time of 60 seconds per point, energy of $6 \mathrm{~J}$ per point, energy density of $193.5 \mathrm{~J} / \mathrm{cm}^{2}$.per point, keeping the stationary tip in contact with the skin at an angle of $90^{\circ}$ and slight pressure. A new test with the same parameters described above followed. To perform the tests, the patient remained seated at an angle of $90^{\circ}$ between trunk and hip and with the leg attached to the seat by means of a brace. The patient was verbally encouraged to maintain maximum strength throughout the exercises (Table 1).

All measures were submitted to the Kruskal-Wallis test and, in case of statistical significance, validated by the Student-Newman-Keuls test for difference of posts (Figure 1).

\section{Results}

Table 2 shows the results of each session in terms of average for extensor muscles with and without laser.

Table 3 shows the results of each session in terms of average for flexor muscles with and without laser.

Table 4 is the values comparing the tests applied on different occasions.

Table 5 shows the mean power values per repetition (PMR) in the four tests performed and their variation.

Figure 2 and Figure 3 show peak torque and average repeat power curves before and after laser application.

\section{Discussion}

The dropouts of using the prosthesis in amputees is high, around $33 \%$ and the return to work is around $10 \%$ mainly in elderly patients [5]. One of the predominant factors for this is muscle fatigue due to exertion, which justifies the search for methods that improve performance. Subjecting the muscles to overload above the usual for it causes there to be breakage 
Citation: Plapler H, Sugawara AT, Montelli RC, et al. (2020) Low Intensity Laser as an Adjuvant to Improve Muscle Strength after Exercise: A Case Report. J Phys Med 2(1):55-59

Table 2: Peak torque (PT) and total work performed (TWP) of the extensor muscles in the 10 sessions (N. m).

\begin{tabular}{|l|l|l|l|l|}
\hline \multicolumn{2}{|l|}{ No laser } & \multicolumn{2}{l|}{ With laser } \\
\hline Session & PT & TWP & PT & TWP \\
\hline 1 & 128.82 & 6428.796 & 142.380 & 6587.448 \\
\hline 2 & 128.82 & 7041.708 & 139.668 & 7089.167 \\
\hline 3 & 131.532 & 6750.167 & 136.956 & 6916.956 \\
\hline 4 & 126.108 & 6945.432 & 134.244 & 7485.120 \\
\hline 5 & 138.312 & 7258.668 & 147.804 & 7735.980 \\
\hline 6 & 130.176 & 7556.988 & 146.448 & 7626.144 \\
\hline 7 & 143.736 & 6889.835 & 138.312 & 7434.948 \\
\hline 8 & 143.736 & 7131.204 & 145.092 & 7959.720 \\
\hline 9 & 136.956 & 7342.740 & 145.092 & 7428.167 \\
\hline 10 & 134.244 & 7685.809 & 151.872 & 8009.892 \\
\hline
\end{tabular}

For PT

$\mathrm{H}=7.2341$

Degrees of freedom $=1$

(p) Kruskal-Wallis $=0.0072$

Student-Newman-Keuls

Groups $(1$ and 2$)=$
Dif. Posts p-value

7.1000 $\quad 0.0073$

For TWP

$\mathrm{H}=2.7657$

Degrees of freedom $=1$

(p) Kruskal-Wallis $=0.0963$

Table 3: Peak torque (PT) and total work performed (TWP) of the flexor muscles in the 10 sessions (N. m)

\begin{tabular}{|l|l|l|l|l|}
\hline \multicolumn{2}{|l|}{ No laser } & \multicolumn{2}{l|}{ With laser } \\
\hline Session & PT & TWP & PT & TWP \\
\hline 1 & 89.495 & 6138.612 & 97.632 & 6601.008 \\
\hline 2 & 97.632 & 7098.660 & 105.768 & 7341.384 \\
\hline 3 & 93.564 & 6474.899 & 107.124 & 7132.560 \\
\hline 4 & 86.784 & 7254.600 & 96.275 & 6832.883 \\
\hline 5 & 101.700 & 7363.079 & 105.768 & 7064.760 \\
\hline 6 & 98.988 & 7223.412 & 104.412 & 7009.164 \\
\hline 7 & 105.768 & 6541.344 & 94.920 & 6150.815 \\
\hline 8 & 104.412 & 7269.516 & 105.768 & 6957.636 \\
\hline 9 & 100.344 & 6771.864 & 92.207 & 6286.416 \\
\hline 10 & 98.988 & 6224.040 & 97.632 & 5993.520 \\
\hline
\end{tabular}

For PT

$\mathrm{H}=0.8329$

Degrees of freedom $=1$

(p) Kruskal-Wallis $=0.3614$

\section{For TWP}

\section{$\mathrm{H}=0.3657$}

Degrees of freedom $=1$

(p) Kruskal-Wallis $=0.5453$

Table 4: PT and TWP values (in N .m) in the 4 tests applied and their relative variation (\%) Vel: $60 \%$ s.

\begin{tabular}{|l|l|l|l|l|l|l|l|l|}
\hline & Test & 1 & 2 & Variation 1:2 & 3 & Variation 2:3 & 4 & Variation 3:4 \\
\hline \multirow{2}{*}{ Extensors } & PT & 132 & 138 & $5 \%$ & 140 & $0 \%$ & 151 & $8 \%$ \\
\cline { 2 - 9 } & TTR & 1516 & 1557 & $3 \%$ & 1406 & $-10 \%$ & 1654 & $18 \%$ \\
\hline \multirow{2}{*}{ Flexors } & PT & 91 & 98 & $7 \%$ & 111 & $11 \%$ & 99 & $-11 \%$ \\
\cline { 2 - 9 } & TTR & 1470 & 1427 & $-3 \%$ & 1395 & $-2 \%$ & 1630 & $17 \%$ \\
\hline
\end{tabular}

For extensors

$\mathrm{H}=4.3548$

Degrees of freedom $=1$

(p) Kruskal-Wallis $=0.0369$

Student-Newman-Keuls comparisons Dif. Posts

Groups (1 and 2) =

\section{For flexors}

$\mathrm{H}=0.4839$

Degrees of freedom $=1$

(p) Kruskal-Wallis $=0.4867$ 
Citation: Plapler H, Sugawara AT, Montelli RC, et al. (2020) Low Intensity Laser as an Adjuvant to Improve Muscle Strength after Exercise: A Case Report. J Phys Med 2(1):55-59

Table 5: Average power values per repetition (in W) in the 4 tests applied and their relative variation (\%) Vel: $300 \%$ s.

\begin{tabular}{|l|l|l|l|l|l|l|l|l|}
\hline & Test & $\mathbf{1}$ & $\mathbf{2}$ & Variation 1:2 & $\mathbf{3}$ & Variation 2:3 & $\mathbf{4}$ & Variation 3:4 \\
\hline Extensors & & 173 & 166 & $-4 \%$ & 171 & $3 \%$ & 208 & $22 \%$ \\
\hline Flexors & & 140 & 125 & $-11 \%$ & 143 & $14 \%$ & 149 & $4 \%$ \\
\hline
\end{tabular}

For extensors

$\mathrm{H}=4.3548$

Degrees of freedom $=1$

(p) Kruskal-Wallis $=0.0369$

Student-Newman-Keuls

Groups $(5$ and 6$)=$
Dif. Posts

3.0000
For flexors

$\mathrm{H}=4.3548$

Degrees of freedom $=1$

(p) Kruskal-Wallis $=0.0369$

Student-Newman-Keuls

Groups $(7$ and 8$)=$
Dif post $\mathrm{p}$-value

3.00000 .0495
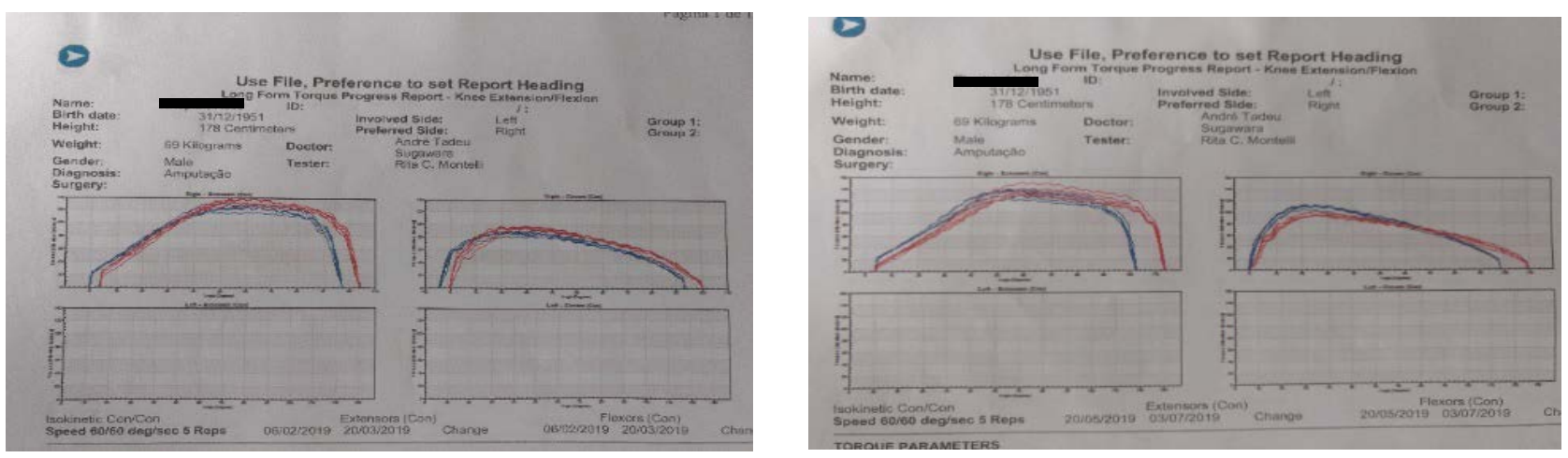

Figure 2: Comparison of PT variation after exercise (left) and after exercise + laser application (right).
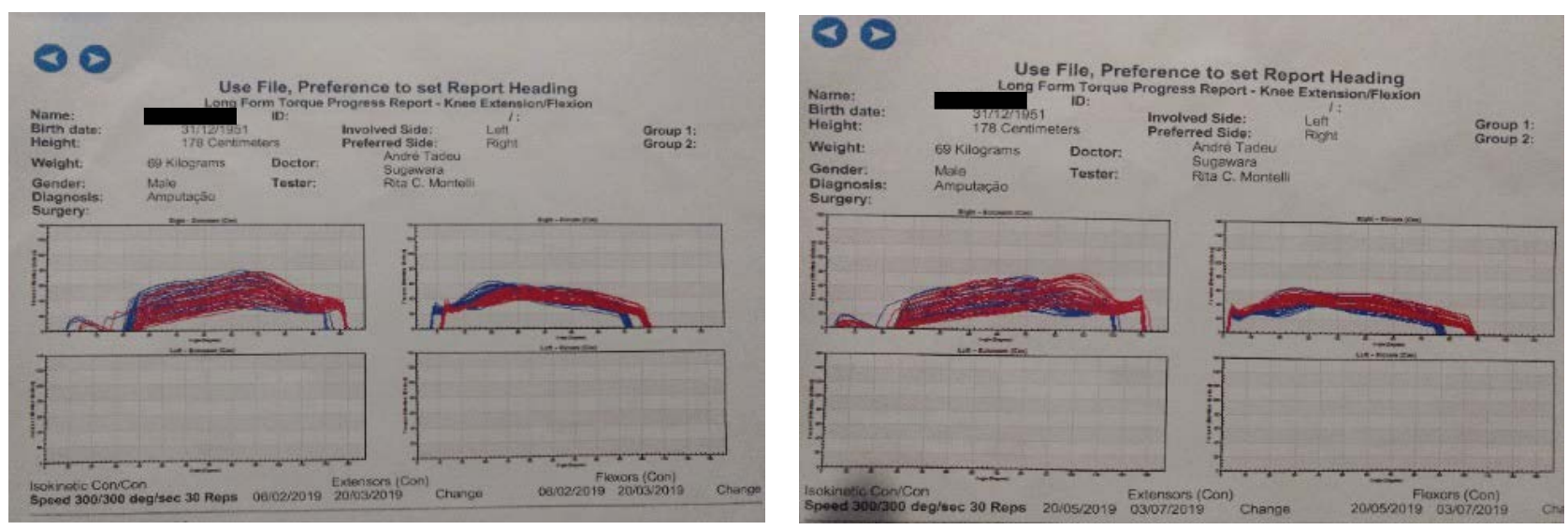

Figure 3: Comparison of the variation of ARP after exercise (left) and after exercise + laser application (right).

of homeostasis and causes the changes that lead to adaptation [6]. The isokinetic exercise is one in which the fixed parameter is the angular velocity of movement, working a single joint at a time. With low angular speed the request for force is very large throughout the arc of motion, allowing for greater gains [7]. The concentric contraction occurs when the device exerts a resistance against the patient's effort. The eccentric contraction is that an external force is applied with increased muscle tension during its stretching, that is, a resistance is produced against the force generated by the device. This type of exercise promotes a lower recruitment of motor units and lower energy expenditure with consequent increase in the strength produced by the muscle. The device (Cybex dynamometer) uses the patient's strength to produce mechani- cal resistance, generating a graph that allows to evaluate the force employed throughout the movement, that is, the force generated by the patient is what determines the force generated by the machine, thus resulting in a maximum exercise throughout the range of motion. This is important because it allows to visualize whether the patient is doing the maximum effort, eliminating possible accommodations during exercise. We found an expected PT increase for the extensor muscles due only to physical activity that can be noticed by the values between the baseline test and the value reached in the second test. The plateau reached between the second and third test indicates the stability of muscle strength achieved by the application of the exercises. It can then be inferred that the dose of $6 \mathrm{~J} /$ point caused an increase in the torque 
of the extensor muscles by the values measured in the last test compared to the third test. It is interesting to note that from the seventh session onwards the PT values for the extensors were consistently higher, indicating a cumulative effect of the laser. It is also possible to notice that $90 \%$ of the values obtained with the laser were higher in relation to the same session without the application of the laser. For flexors, although the PT values were higher with laser in $70 \%$ of sessions, there was no statistical correlation. The laser action occurs, presumably, on the cellular respiratory chain, with increased ATP production. Currently it is speculated that this is due to the expansion of the volume and alteration of the viscosity of the water layer next to the cell and mitochondrial membrane [8]. One of the causes of laser failure in the flexor muscles regarding the PT may be the greater amount of fat on the posterior side of the thigh that would cause greater spreading and lower absorption of the laser in this region [9]. Regarding to TWP, the $10 \%$ drop in the third test shows that the exercise caused fatigue and that the LBI was able to reverse this process with an increase of $18 \%$ for the extensors and $17 \%$ for the flexors. Thus, it is necessary to evaluate what would cause better muscle performance without increasing the maximum strength. This can be explained by the increase in average power per repetition (APR), which was shown to be higher with the use of laser for both extensors and flexors, indicating that the muscle resisted fatigue for longer. In a controlled clinical trial, muscle recruitment was maintained for longer with the use of LIL compared to placebo [10]. The use of LIL was also able to increase PT and increase the onset of fatigue time in a patient with spasticity due to stroke, with decreased lactate in the blood [11]. However, because it is only one patient, other variables should be considered. In our study, even if the ambient temperature was controlled and constant, the tests were performed without a period of acclimatization of the patient in relation to the external environment, which could influence the initial state of the musculature. Also, this is not a blind study, because the patient knew the protocol, although the device tends to correct this bias. This study shows the action of LIL in increasing peak torque and total work performed and indicates that this treatment, with properly adjusted protocols, can help a program of muscle strengthening and reduction of fatigue in prosthetic patients.

\section{References}

1. Nethery D, Callahan LA, Stofan D, et al. (2000) PLA(2) dependence of diaphragm mitochondrial formation of reactive oxygen species. J Appl Physiol (1985) 89: 72-80.

2. Baroni BM, Leal ECP Jr, Marchi T, et al. (2010) Low level laser therapy before eccentric exercise reduces muscle damage markers in humans. Eur J Appl Physiol 110: 789-796.

3. Leal Junior ECP, Nassar FR, Tomazoni SS, et al. (2000) A laserterapia de baixa potência melhora o desempenho muscular mensurado por dinamometria isocinética em humanos. Fisioterapia e Pesquisa 7: 317-321.

4. Vassao PG, Souza MC, Silva BA, et al. (2019) Photobiomodulation via a cluster device associated with a physical exercise program in the level of pain and muscle strength in middle-aged and older women with knee osteoarthritis: A randomized placebo-controlled trial. Lasers Med Sci 35: 139-148.

5. https://cdn.publisher.gn1.link/actafisiatrica.org.br/pdf/en_ v14n2a05.pdf

6. https://www.fef.unicamp.br/fef/sites/uploads/deafa/qvaf/estrategias_cap8.pdf

7. https://edisciplinas.usp.br/pluginfile.php/5253340/mod_resource/content/1/Apostila\%20-\%20Isocin\%C3\%A9tica\%20-\%20 disciplina\%20RCG0453.pdf

8. Sommer AP (2019) Revisiting the Photon/Cell Interaction Mechanism in Low-Level Light Therapy. Photobiomodul Photomed Laser Surg 37: 336-341.

9. Chavantes MC (2009) Laser na biomedicina: Princípios e prática: Guia para iniciantes. Atheneu: 281.

10. Jorge FS, Azevedo RC (2011) Laserterapia de baixa intensidade e recuperação muscular em fadiga: Ensaio clínico controlado. Biologia \& Saúde 3: 21-30.

11. Neves M, Reis MCR, Andrade EAF, et al. (2014) Efeito imediato do laser de baixa intensidade sobre o músculo espástico fatigado: Estudo de caso. XXIV Congresso Brasileiro de Engenharia Biomédica - CBEB 2014: 1337-1340.

DOI: $10.36959 / 942 / 340$

Copyright: (C) 2020 Plapler $\mathrm{H}$, et al. This is an open-access article distributed under the terms of the Creative Commons Attribution License, which permits unrestricted use, distribution, and reproduction in any medium, provided the original author and source are credited. 